\title{
Chronik der Medienentwicklung in Deutschland 2000
}

\section{Christiane Matzen / Anja Herzog}

1. Medienentwicklung europäisch/ international

2. Medienpolitik Bund/Länder

3. Medienrecht

4. Medienkonzerne

4.1 Kirch

4.2 Bertelsmann
5. Marktanteile/Werbemarkt

6. Öffentlich-rechtlicher Rundfunk einzelne Anstalten

7. Privater Rundfunk

7.1 Landesmedienanstalten

7.2 Private Hörfunkveranstalter

7.3 Private Fernsehveranstalter

\section{Medienentwicklung europäisch / international}

Am 10. Januar kündigen der weltgrößte Online-Dienst America Online (AOL) und der US-Medienkonzern Time Warner ihren Zusammenschluss an. Die größte Fusion aller Zeiten soll im Zuge eines Aktientausches mit einem Volumen von $665 \mathrm{Mrd}$. DM realisiert werden. AOL hatte 1999 einen Jahresumsatz von 9,1 Mrd. DM, Time Warner einen Jahresumsatz von 51,1 Mrd. DM erzielt.

Der bereits über sechs Jahre währende Streit zwischen der EU und den Vertretern des deutschen und österreichischen Buchhandels über die Frage der Buchpreisbindung wird mit einem Schreiben ${ }^{1}$ vom 8. Februar an die Rechtsvertreter des deutschen und österreichischen Buchhandels durch EU-Wettbewerbskommissars Mario Monti beendet. Eine grenzüberschreitende Buchpreisbindung darf es Monti zufolge aus wettbewerbsrechtlichen Gründen nicht geben, rein nationale Regelungen aber sind erlaubt. In Deutschland darf es folglich weiter ein System der Buchpreisbindung geben, die österreichischen Verleger dürfen jedoch nicht in die deutsche Buchpreisbindung einbezogen werden. Verkäufe von Verlagserzeugnissen an Endabnehmer in anderen Mitgliedstaaten der EU unterliegen in keinem Fall der Preisbindung, reine Re-Importe allerdings schon.

Die Kommission der Europäischen Union verabschiedet am 12. Juli die Transparenzrichtlinie $^{2}$, derzufolge öffentliche Unternehmen mit einem Nettojahresumsatz von mehr als 40 Mio. Euro künftig ihre öffentlichen und kommerziellen Aktivitäten buchhalterisch getrennt ausweisen müssen. Die Mitgliedsstaaten müssen die Transparenzrichtlinie bis zum 31. Juli 2001 in nationales Recht umsetzen. Umstritten bleibt zunächst, ob auch die öffentlich-rechtlichen Rundfunkanstalten in den Anwendungsbereich der Richtlinie fallen.

Bei den Wahlen der Internet Corporation for Assigned Names and Numbers (ICANN) wird am 11. Oktober Andy Müller-Maguhn, Journalist und Sprecher des Hamburger Chaos Computer Clubs, als europäischer Vertreter in das Direktorium gewählt. Die 1998 gegründete weltweite Internet-Regierung entscheidet u. a. über die Vergabe der Adress-Endungen .com und .eu. Von den 19 ICANN-Direktorensitzen wer-

1 Abgedruckt in epd medien 11/2000.

2 Richtlinie 2000/52/EG der Kommission vom 26. Juli 2000 zur Änderung der Richtlinie 80/723/EWG über die Transparenz der finanziellen Beziehungen zwischen den Mitgliedstaaten und den öffentlichen Unternehmen, Amtsblatt der Europäischen Gemeinschaften 29.7.2000 L $193 / 75$. 
den aufgrund der Wahl der Internet-Nutzer fünf - je einer für die Regionen Afrika, Asien/ Pazifik, Europa, Lateinamerika/Karibik und Nordamerika - besetzt.

Am 13. Dezember unterzeichnen die europäische Fußballunion UEFA und die Europäische Rundfunkunion EBU den Vertrag über die Fernsehübertragungsrechte der Fußballeuropameisterschaft 2004 in Portugal. Die Rechte kosten rund eine Milliarde Mark. Durch den Vertrag ist gesichert, dass die EM-Spiele in Deutschland bei ARD und ZDF im Free-TV zu sehen sein werden.

\section{Medienpolitik Bund / Länder}

Die Kommission zur Ermittlung des Finanzbedarfs der Rundfunkanstalten (KEF) legt am 11. Januar ihren Bericht vor. Die Kommission schlägt vor, die monatliche Rundfunkgebühr ab 1. Januar $2001 \mathrm{zu}$ erhöhen (siehe unten).

Bundesjustizministerin Herta Däubler-Gmelin kündigt am 18. Januar an, das Zeugnisverweigerungsrecht der Presse ausweiten zu wollen. Das Recht soll künftig auch selbstrecherchiertes Material umfassen ${ }^{3}$.

Am 15. Juni billigen die Ministerpräsidenten der Bundesländer einen Entwurf des Fünften Rundfunkänderungsstaatsvertrags ${ }^{4}$, der zum 1. Januar 2001 in Kraft treten soll und unter anderem vorsieht, Werbung im Videotext von ARD und ZDF zu verbieten sowie privaten Regionalfernsehsendern nach Maßgabe des zuständigen Bundeslandes großzügige Werbemöglichkeiten zu erlauben. Beschlossen wird außerdem eine Erhöhung der monatlichen Rundfunkgebühr ab 1. Januar 2001 um 3,33 DM auf 31,58 DM. Mit dem Gebührenbeschluss folgen die Regierungschefs dem Votum ihrer Rundfunkkommission, die wiederum einem Vorschlag der KEF folgte. Von den monatlich fälligen 31,58 DM gehen 22,32 DM an die ARD, 7,84 DM an das ZDF, beide müssen hiervon jedoch 0,315 DM (zusammen also 0,63 DM) an den Kulturkanal Arte weiterleiten. Das Deutschlandradio bekommt 0,79 DM, 0,63 DM gehen an die Landesmedienanstalten.

Nach ihren 1999er Sparerlassen für die Deutsche Welle (DW) konkretisiert die rotgrüne Bundesregierung im Juni ihre Vorstellungen für eine zukunftsorientierte Weiterentwicklung des Auslandsrundfunks. Dabei zielt sie auf einen Ausbau der OnlineDienstleistungen, eine Neuausrichtung des Fernsehprogramms auf Kultur sowie eine politische Koordination der Radioprogramme. Bei allem wird eine enge Zusammenarbeit mit den Ländern sowie die Einbindung der ARD-Landesrundfunkanstalten sowie des ZDF angestrebt. Die Deutsche Welle solle künftig in der Lage sein, die gesellschaftspolitischen oder auch wirtschaftlichen Informationsbedürfnisse bestimmter Zielgruppen „schnell und punktgenau zu bedienen“. Als technischer Verteilweg hierfür wird das Internet bevorzugt (und weniger der klassische Rundfunk über erdgebundene Wellen oder Satellit). Über das Online-Medium soll sich jeder Interessent Text-, Bildund Multimedia-Angebote bei der DW abrufen können, so das Zukunftsmodell. Die Rundfunkreferenten der Länder beschäftigen sich am 27. Juli in Berlin mit den „Überlegungen zur Neugestaltung des Deutschen Auslandsrundfunks“. Auf der Jahreskonferenz der Ministerpräsidenten vom 25. bis 27. Oktober in Schwerin werden vier Länder (Bayern, Berlin, Nordrhein-Westfalen, Rheinland-Pfalz) beauftragt, auf politischer Ebene - nicht wie ursprünglich geplant auf Fachebene - mit der Bundesregierung

3 Gesetzentwurf der Bundesregierung s. BR-Drs. 441/00.

4 Abgedruckt in epd medien 57/2000. 
über das Konzept des Bundes zur Zukunft des Auslandsrundfunks zu beraten. (s. auch unter 6.)

Bundeswirtschaftsminister Werner Müller legt Ende September unter dem Titel „Startszenario 2000 - Sachstandsbericht und Empfehlungen zur Digitalisierung von Hörfunk und Fernsehen“ 5 einen Bericht der Arbeitsgruppe „Initiative Digitaler Rundfunk“ (IDR) vor. Die IDR soll die Umstellung der Rundfunkübertragung in Deutschland von analoger auf digitale Technik bis zum Jahr 2010 vorbereiten. In der IDR wirken die Länder, die Radio- und Fernsehsender sowie ihre Interessenverbände und Rundfunktechniker und -wissenschaftler mit. Vor allem die Privatsender hatten dazu aufgefordert, den Normwechsel in der Sendetechnik schon vor $2010 \mathrm{zu}$ vollenden, da die so genannte Simulcast-Phase, also die Übergangsphase, während derer sowohl ana$\log$ als auch digital auszustrahlen ist, zu teuer sei.

Die 1997 von den Ländern eingerichtete Kommission zur Ermittlung der Konzentration im Medienbereich (KEK) veröffentlicht am 28. November erstmals einen Konzentrationsbericht ${ }^{6}$, der laut Rundfunkstaatsvertrag alle drei Jahre von den Landesmedienanstalten vorgelegt werden soll. Der Bericht betrachtet neben dem Fernsehen auch die Konzentration im Hörfunk, beleuchtet Verflechtungen zwischen Rundfunk und Presse und spricht von „hochkonzentrierten Strukturen“ auf den Medienmärkten.

In einem gemeinsamen Schreiben vom 7. Dezember lehnen die öffentlich-rechtlichen und privaten Sender die geplante Änderung des Urheberrechts ab, die auf eine Stärkung der vertraglichen Stellung von Urhebern und ausübenden Künstlern zielt. Sie befürchten massive Nachteile für die deutsche Produktionswirtschaft. Das Vorgehen trifft auf die entschiedene Kritik der IG Medien.

\section{Medienrecht}

Im Streit zwischen RTL und dem Anbieter des Werbeblockers „Fernsehfee“ untersagt das Berliner Landgericht der TC Unterhaltungselektronik AG, ein Fernsehgerät bei Werbesendungen im laufenden Programm durch die so genannte „Fernsehfee“ automatisch auf einen anderen zur gleichen Zeit werbefreien Kanal umzuschalten. Das im Februar bekannt werdende Urteil stammt bereits vom 7. Dezember 1999. Ein Urteil des Berliner Kammergerichts vom 22. Oktober 1999 hingegen hatte es der TC Unterhaltungselektronik gestattet, Signale auszustrahlen, die den Videorekorder bei der Aufzeichnung anhalten.

Am 10. Februar verabschieden die Landesmedienanstalten neue Werberichtlinien ${ }^{7}$ für den privaten Rundfunk; sie treten am 29. Mai in Kraft. Die Bestimmungen regeln u. a. das Split-Screen-Verfahren und die virtuelle Werbung und konkretisieren damit

5 Abgedruckt in epd medien 92/2000.

6 Vgl. www.kek-online.de/kek/information/publikation/mk-bericht/index.html; außerdem in Auszügen abgedruckt in epd medien 99/2000.

7 „Gemeinsame Richtlinien der Landesmedienanstalten für die Werbung, zur Durchführung der Trennung von Werbung und Programm und für das Sponsoring im Fernsehen in der Neufassung vom 10.2.2000“ sowie "Gemeinsame Richtlinien der Landesmedienanstalten für die Werbung, zur Durchführung der Trennung von Werbung und Programm und für das Sponsoring im Hörfunk in der Neufassung vom 10.2.2000", abgedruckt in Funkkorrespondenz Nr. $16-17 /$ Beilage. 
die im April in Kraft tretenden Bestimmungen des 4. Rundfunkänderungsstaatsvertrags.

Der Landtag in Schwerin verabschiedet am 15. März das novellierte Landesrundfunkgesetz Mecklenburg-Vorpommern (RundfG-M-V) ${ }^{8}$, das am 1. April in Kraft tritt. Private Rundfunkveranstalter mit Sitz in Mecklenburg-Vorpommern dürfen danach keine Wahlwerbung von politischen Parteien mehr ausstrahlen. Außerdem ist nach dem neuen Gesetz erstmals kommerzielles Lokal- und Regionalfernsehen möglich, während privates kommerzielles Lokalradio auch künftig nicht erlaubt ist.

Am 1. April tritt der 4. Rundfunkänderungsstaatsvertrag ${ }^{9}$ in Kraft. Die Novelle setzt vor allem die Regelungen der EU-Fernsehrichtlinie vom Juni 1997 in deutsches Recht um und berechtigt u. a. die öffentlich-rechtlichen Veranstalter, ihre gesetzlich bestimmten Programme auch in digitaler Technik zu verbreiten sowie zusätzliche, ihrem Programmauftrag entsprechende digitale Angebote zu veranstalten. Zudem wird die im Vorjahr beschlossene Must-Carry-Lösung für digitalisierte Kabelanlagen, also die Zuweisung von insgesamt drei bundesweiten Kabelkanälen für ARD Digital und ZDFVision, gesetzlich festgeschrieben. Die Novelle regelt auch den chancengleichen und diskriminierungsfreien Zugang aller digitalen Fernsehveranstalter zu den multimedialen Betriebsplattformen und enthält neue Vorschriften für den Bereich Jugendschutz im digitalen Fernsehen sowie großzügigere Werberegelungen. Außerdem sichert der neue Rundfunkstaatsvertrag die Live-Übertragung von wichtigen Großereignissen im Free-TV.

Das Oberlandesgericht Köln entscheidet am 9. Juni, dass das Gratis-Blatt „20 Minuten Köln“, das der norwegische Konzern Schibsted seit 13. Dezember 1999 herausbringt, weiter erscheinen darf. Der Kölner Verlag DuMont-Schauberg als Kläger habe den notwendigen Nachweis einer „Vernichtungskampagne“ gegen die von ihm verlegten kostenpflichtigen Blätter („Kölner Stadt-Anzeiger“, „Express“) nicht erbracht. ${ }^{10}$ Zuvor hatte bereits das Oberlandesgericht Karlsruhe am 18. Mai entschieden, diese Vertriebsart verstoße nicht gegen den Wettbewerb. ${ }^{11}$ In diesem Zusammenhang diskutierte der in Freiburg ansässige 4. Zivilsenat des OLG Karlsruhe auch die Frage, ob aus einer reinen Anzeigenfinanzierung von Zeitungen Gefahren für die Pressefreiheit und im Besonderen für die Unabhängigkeit der Berichterstattung resultieren, und verneinte dies. Da beide unterlegenen Verlage, der Axel-Springer-Verlag in Freiburg und der Verlag DuMont in Köln, daraufhin ankündigen, in die Revision zum Bundesgerichtshof gehen zu wollen, wird mit einer höchstrichterlichen Sammelentscheidung in der Frage der wettbewerblichen Zulässigkeit kostenloser Presseprodukte gerechnet.

Der sachsen-anhaltinische Landtag verabschiedet am 22. Juni ein neues Mediengesetz, das das Privatrundfunkgesetz für Sachsen-Anhalt ablöst. Es sieht den Umstieg auf digitale Übertragungstechniken für Radio und Fernsehen bis spätestens 2010 vor. Weiterhin ermöglicht das Gesetz, dass private Hörfunkveranstalter ab August 2001 auch regionale Werbung ausstrahlen können. Der Landesrundfunkausschuss erhält den Namen „Medienanstalt Sachsen-Anhalt (MSA)“.

Am 26. Juni beschließt die DLM die neue „Satzung über die Zugangsfreiheit zu digitalen Diensten gemäß $\int 53$ Abs. 7 Rundfunkstaatsvertrag“. Die Bestimmungen umfas-

8 Abgedruckt in epd medien 33/2000 und Funkkorrespondenz Nr. 18/19/Bl.

9 Abgedruckt in epd medien 36/1999 und Funkkorrespondenz Nr. 52/1999.

10 Az. 6 U 40/00, Urteil dokumentiert in epd medien Nr. 57/2000.

11 Az. 4 U 46/99, Urteil dokumentiert in epd medien Nr. 57/2000. 
sen die inhaltliche und verfahrensmäßige Konkretisierung der chancengleichen und angemessenen Zugangsbedingungen zu digitalen Diensten. ${ }^{12}$

$\mathrm{Ab}$ 1. Juli gilt eine neue Jugendschutzsatzung für digitale TV-Programme, die festlegt, welche Anforderungen an die Verschlüsselung und Vorsperrung von Sendungen im digitalen Fernsehen zu stellen sind. Gelockerte Zeitgrenzen gelten danach nur, wenn der Anbieter der Sendungen sicherstellt, dass die Vorsperrung lediglich für die Dauer dieser Sendung durch Eingabe eines vierstelligen persönlichen Jugendschutz-Codes des Nutzers freigeschaltet werden kann.

Das Verwaltungsgericht Köln weist am 29. Oktober die Klage von ARD und ZDF gegen die Regulierungsbehörde für Telekommunikation und Post (RegTP) aus formalen Gründen zurück. ARD und ZDF hatten sich gegen einen Beschluss der RegTP gewandt, demzufolge die Telekom alle Fernsehveranstalter bei den Gebühren für die Kabeleinspeisung gleich (diskriminierungsfrei) zu behandeln habe; die Telekom hatte daraufhin Gebühren für die Einspeisung der bislang kostenlos über Kabel verbreiteten öffentlichrechtlichen Programme gefordert.

Am 29. November untersagt das Landgericht Leipzig dem in Mainz ansässigen Kabelnetzbetreiber Primacom, die TV-Programme Pro Sieben, Kabel 1, TM3 und Deutsches Sportfernsehen ausschließlich digital und kostenpflichtig zu verbreiten. Fernsehveranstalter hätten als Urheber alleine darüber zu entscheiden, welche Kabelnetzbetreiber ihre Programme zu welchen Bedingungen in welche Netze einspeisen.

Am 19. Dezember verabschiedet der hessische Landtag zwei novellierte Rundfunkgesetze, die zum Januar 2001 in Kraft treten sollen. Der Hessische Rundfunk (HR) wird der Novelle zum HR-Gesetz zufolge künftig 28 statt 16 Rundfunkratsmitglieder haben, die von einer Reihe neuer Organisationen entsandt werden, so dass die gesellschaftlichen Verhältnisse besser widergespiegelt seien. Die von CDU und FDP initiierte Novellierung war in den vergangenen Monaten stark als Versuch kritisiert worden, den Parteien Einfluss beim öffentlich-rechtlichen Rundfunk zu sichern. Die Neufassung des Hessischen Privatrundfunkgesetzes (HPRG) erlaubt nun auch Ballungsraumfernsehen und den Ausbau des Kabelnetzes auf 150 Programme.

\section{Medienkonzerne}

\subsection{Kirch}

Die Hamburgische Anstalt für neue Medien (HAM) genehmigt am 29. Januar die Übernahme von 59,8 Prozent der Anteile am Hamburger TV-Ballungsraumsender „Hamburg 1" durch die Kirch-Gruppe. Weitere Gesellschafter bleiben die Deutsche Fernsehnachrichten GmbH (DFA) mit 29,17 Prozent und die Axel-Springer-Verlag AG mit 11,2 Prozent.

Am 14. April teilt die Kirch-Gruppe mit, dass die Verträge über den Einstieg Rupert Murdochs bei der Kirch-Gruppe unterzeichnet worden sind. Für 2,9 Mrd. DM steigt Murdoch über sein Unternehmen British Sky Broadcasting Group (BSkyB) mit 24 Prozent bei der Kirch Pay-TV GmbH \& Co. KGaA ein. Am 13. Juni legt die ARD beim Europäischen Gerichtshof Klage gegen die Entscheidung der EU-Kommission vom 21. März ein, in der die wechselseitigen Beteiligungen der Pay-TV-Sender BSkyB und Premiere World genehmigt worden war. Die ARD befürchtet eine marktbeherrschende

12 Vgl. die Satzung unter www.alm.de. 
Stellung der Allianz, obwohl die EU-Kommission Kirch und Murdoch Auflagen erteilt hatte, um Wettbewerbsbedenken und Zugangsschranken für andere Anbieter auszuräumen.

Am 29. April teilen die Kirch-Gruppe und der Deutsche Fußballbund (DFB) mit, dass die Fernsehrechte der Fußballbundesliga für vier Jahre erneut an Kirch gehen. Der Kaufpreis beträgt die bislang einmalige Rekordsumme von insgesamt $3 \mathrm{Mrd}$. DM und hat sich damit gegenüber dem letzten Fernsehvertrag mehr als verdoppelt.

Mit der Zustimmung der Aktionäre der Pro Sieben Media AG am 22. August wird die Fusion von Pro Sieben und SAT.1 besiegelt. Durch die Verschmelzung der beiden Sender entsteht das größte deutsche private Fernsehunternehmen mit einem gemeinsamen Umsatz von rund $2 \mathrm{Mrd}$. Euro. An dem neuen Konzern ist die Kirch Media KGaA mit 52,25 Prozent beteiligt, 11,48 Prozent hält der Axel-Springer-Verlag.

Die Kirch Pay-TV teilt am 31. August ihren Ausstieg aus BSkyB mit, sie verkaufe ihren Anteil am britischen Pay-TV-Anbieter an das Bankenkonsortium Crédit Suisse First Boston (Europe) und an Goldman Sachs International. Die Transaktion sei ein „Element im Rahmen der Gesamtfinanzierung des Kirch Pay-TV“.

Durch den Einstieg zweier neuer Minderheitengesellschafter erhält die Kirch Pay-TV fast 800 Mio. DM an neuem Geld. Am 8. September teilt das Unternehmen mit, dass die Kingdom Holdings 8 B. V. und Capital Research and Management Funds Gesellschafter mit Anteilen von 3,2 \% (346,6 Mio. DM) bzw. 2,76 \% (450 Mio. DM) werden. Beide Investoren sind bereits an der KirchMedia GmbH \& Co KG, dem Unternehmen der KirchGruppe für Free-TV, beteiligt. Weitere 400 Mio. DM (2,4 \%) investiert - laut Pressemitteilung vom 21. September - die Lehman Brothers Merchant Banking II L.P. bei der Kirch Pay-TV, auch Lehman Brothers ist bereits mit 2,76 \% an KirchMedia beteiligt.

In ihrer Sitzung vom 24. Oktober erklärt die Kommission zur Ermittlung der Konzentration im Medienbereich (KEK) die neue Senderfamilie der Kirch-Gruppe mit den Fernsehprogrammen SAT.1, Pro Sieben, Kabel 1 und N 24 konzentrationsrechtlich für unbedenklich. Im prüfungsrelevanten Zeitraum von September 1999 bis August 2000 erzielten die vier Kirch-Sender einen Zuschaueranteil von zusammen 26,39 Prozent. Vorherrschende Meinungsmacht wird laut Rundfunkstaatsvertrag ab einem Marktanteil von 30 Prozent (bzw. geringfügiger Unterschreitung dieses Anteils) vermutet; erst dann ist ein Einschreiten Pflicht.

\subsection{Bertelsmann}

Die EU-Kommission stimmt am 21. März der neuen Eigentumsstruktur beim Fernsehsender Vox zu. Sie sehe keine Wettbewerbsbedenken gegen den Kauf von 49,9 Prozent der Vox-Anteile durch die CLT-Ufa (Bertelsmann), da sich der Marktanteil der CLTUfa-Senderfamilie dadurch nicht unzulässig vergrößern werde. Auch die deutsche Kommission zur Ermittlung der Konzentration im Medienbereich (KEK) teilt am 23. März mit, dass sie keine medienkonzentrationsrechtlichen Bedenken hege. Die CLTUfa hatte Ende 1999 die 49,9 Prozentanteile von Rupert Murdoch für rund 600 Mio. DM erworben.

Am 7. April teilt der Bertelsmann-Konzern mit, dass die CLT-Ufa mit dem britischen Fernsehproduzenten Pearson TV fusionieren werde. Unter dem Namen RTL Group entsteht damit Europas größtes Rundfunkunternehmen. CLT-Ufa und Person TV erzielten 1999 bei einem addierten Umsatz von rund 7,4 Mrd. DM einen Vorsteuergewinn von knapp 900 Mio. DM. Die EU-Kommission teilt am 30. Juni mit, dass sie die Fusion 
genehmige, da keine spürbaren negativen Auswirkungen auf den Wettbewerb zu befürchten seien. Auch die KEK erklärt die Fusion am 19. September für unbedenklich.

Vor allem wegen stark gestiegener Anzeigenerlöse kann Gruner + Jahr im Geschäftsjahr 1999/2000 seinen Umsatz um 6,9 Prozent auf 5,76 Milliarden Mark steigern. Wie das von Bertelsmann beherrschte Unternehmen am 31. August mitteilt, habe die Umsatzrendite ohne die Einflüsse außerordentlicher Aufwendungen oder Erträge bei 10,5 Prozent gelegen. Das um 23,1 Prozent gesteigerte Ergebnis (vor Zinsen und Steuern) von 751 Mio. DM sei der „Zweitbeste“ Wert in der Unternehmensgeschichte. Das Unternehmen erwirtschaftete zuletzt 58 Prozent seines Umsatzes im Ausland, das Gros davon in den Vereinigten Staaten und in Frankreich. Im abgelaufenen Geschäftsjahr hatte es zwei Neueinführungen gegeben: die Einführung der „Financial Times Deutschland“ im Februar und den europaweiten Start von „National Geographic“, das knapp eine Million Leser erreichte. Die „Financial Times Deutschland“ habe innerhalb von fünf Monaten eine verkaufte Auflage von 57.400 Exemplaren erreicht, 120.000 Exemplare seien für das Überschreiten der Gewinnschwelle veranschlagt worden. Die Abonnementauflage der Wirtschaftszeitung beträgt Ende August 20.000 Exemplare.

Am 13. September legt der Bertelsmann-Vorstandsvorsitzende Thomas Middelhoff die Bilanz für das Geschäftsjahr 1999/2000 vor. Danach wurde der Jahresüberschuss der Bertelsmann AG um 45 Prozent gesteigert, der Gewinn betrug 1,3 Mrd. DM bei einem Umsatz von 32,4 Mrd. DM (plus $25 \%$ ).

\section{Marktanteile/Werbemarkt}

Die Fernsehprogramme „Das Erste“ und RTL sind im Jahr 2000 die meistgesehenen Programme mit identischen Marktanteilszahlen. RTL, populärster Sender in den Jahren 1993 bis 1997 und 1999, und die ARD, zuletzt 1998 Marktführer, kommen laut GfKZuschauerforschung auf jeweils 14,3 Prozent Marktanteil (alle Zuschauer ab 3 Jahre, rund um die Uhr). Insgesamt liegen die Marktanteile im Fernsehbereich auf annähernd dem gleichen Niveau wie im Vorjahr, bezogen auf alle Zuschauer bleiben alle Veränderungen unter der Grenze von einem Prozentpunkt. Am stärksten legt der „Big Brother“Sender RTL II zu: Er steigerte seinen Marktanteil um 0,8 Prozentpunkte auf 4,8 Prozent (alle Zuschauer ab 3 Jahre). Die größten Einbußen erleidet SAT.1, das nach den Verlusten von jeweils einem Prozentpunkt in den Jahren 1998 und 1999 nun 0,6 Prozentpunkte abgibt und auf 10,2 Prozent Marktanteil kommt. Die beiden öffentlich-rechtlichen Hauptsender Das Erste und ZDF legen um jeweils 0,1 Prozentpunkte zu; sie profitieren unter anderem von ihren Übertragungen der Spiele der Fußball-Europameisterschaft (das Finale Frankreich - Italien in der ARD ist die meistgesehene Sendung des Jahres). Die Rangfolge der meistgesehenen Sender im Jahr 2000 sieht folgendermaßen aus (in Klammern jeweils die Veränderungen gegenüber 1999): ARD (+0,1 Prozentpunkt) und RTL $(-0,5)$ mit jeweils 14,3 Prozent, ZDF 13,3 (+0,1); ARD-Dritte 12,7 $(+0,2)$; SAT. 1 mit 10,2 Prozent (-0,6); Pro Sieben 8,2 (-0,2); Kabel 1 mit 5,5 Prozent $(+0,1)$; RTL II mit 4,8 Prozent $(+0,8)$; VOX und Super RTL mit jeweils 2,8 Prozent (beide \pm 0 ), Kinderkanal und DSF mit jeweils 1,2 Prozent (beide $-0,1)$; TM3 $( \pm 0)$ und Eurosport $(-0,1)$ mit jeweils 1 Prozent; 3sat 0,9 ( \pm 0$)$; n-tv 0,7 $( \pm 0)$; Phoenix 0,4 (erstmals ausgewiesen); ARTE 0,3 Prozent. Zu berücksichtigen ist beim Vergleich der Zahlen, dass die technischen Reichweiten und Sendezeiten der Programme differieren: ARTE kommt so in seiner Sendezeit von 19 bis 3 Uhr auf einen Marktanteil von 0,6 Prozent (+0,1 gegenüber 1999). Der ARD/ZDF-Kinderkanal erzielt in der Sendzeit von 6 bis 19 Uhr bei den 3- bis 13-Jährigen einen Marktanteil von 15,9 Prozent (-2,3). 
Betrachtet man nur die als werberelevant angesehene Gruppe der 14- bis 49-jährigen Zuschauer, so kommen lediglich die drei großen Privatsender RTL, Pro Sieben und SAT.1 auf zweistellige Marktanteilszahlen: RTL 17,3 Prozent (-0,5); Pro Sieben 13,3 $(-0,2)$; SAT.1 mit 12 Prozent (-0,8), ARD 9,6 (-0,2); ZDF 8,2 ( \pm 0$)$; ARD-Dritte 7,9 $(-0,2)$; RTL II mit 7,1 Prozent $(+1,4)$; Kabel 1 mit 5,4 (+0,1). Umgekehrt liegen die Öffentlich-Rechtlichen in der Gruppe der Zuschauer ab 50 Jahren bei steigenden Marktanteilen deutlich vor den Privaten: ARD 19,4 (+0,4); ZDF 18,7 (+0,2); ARD-Dritte 17,9 $(+0,4)$; RTL 11,8 (-0,6); SAT.1 mit 9,0 (-0,5); Kabel 1 mit 6,1 Prozent $(+0,2)$; Pro Sieben $3,6(-0,2)$; VOX mit 2,0 Prozent $(-0,1)$.

Die tägliche Sehdauer steigt im Jahre 2000 um fünf Minuten auf 190 Minuten, während sie im Jahr davor um drei Minuten gesunken war.

Die Media-Analyse 2000 Radio wird erstmals auf der Basis von Telefoninterviews (Computer Assisted Telephone Interviewing - CATI) durchgeführt. Die als Vollerhebung konzipierte Reichweiten-Studie erfasst das gesamte in der Bundesrepublik empfangbare Radioangebot. Die Gesamtzahl beträgt 261 Sender, davon als Werbeträger ausgewiesen sind 75 Hörfunksender/-programme und 66 Werbefunkkombinationen. Befragt wurden insgesamt 57273 Personen in 111 Teilgebieten.

Mit der Fusion des SAT.1-Vermarkters Media 1 und des Pro Sieben-Vermarkters Media Gruppe München (MGM) zu Seven One Media nimmt am 15. November Europas größtes TV-Vermarktungsunternehmen sein Geschäft auf. Laut Seven One Media erzielte das fusionierte Unternehmen 1999 einen addierten Netto-Umsatz von 3,9 Mrd. DM (Publitalia/Mediaset: 3,6 Mrd. DM; IP Deutschland: 3,4 Mrd. DM).

\section{6. Öffentlich-rechtlicher Rundfunk - einzelne Anstalten}

Ab dem 1. April wird das digitale Jugendradio „Das Ding“ des Südwestrundfunks auf UKW verbreitet. Da das Programm mangels vorhandener Digitalempfangsgeräte nur von wenigen empfangen werden kann, wird es auch über das Internet ausgestrahlt.

„ZDF Doku“ geht ebenfalls am 1. April auf Sendung. Der neue Reportage- und Dokumentationskanal ist neben ZDF Info und dem Theaterkanal das dritte eigenständige und exklusiv für die digitale Welt veranstaltete Fernsehangebot des Zweiten Deutschen Fernsehens.

Ein „10-Punkte-Programm“ zur Zukunft der Deutschen Welle (DW) stellt DW-Intendant Dieter Weirich am 6. April in Köln vor. Es beschreibt, auf welche Weise die DW künftig unter drastisch eingeschränkten finanziellen Rahmenbedingungen ihre Hörfunkund Fernsehprogramme sowie das Internetangebot einsetzen will. Der Etat der DW, der im Mai 1999 noch bei 635 Mio. DM gestanden hatte, sinkt bis 2003 auf 546 Mio. DM.

Am 25. Mai billigt der Rundfunkrat von Radio Bremen (RB) die von RB-Intendant Heinz Glässgen geplanten Strukturveränderungen. Die vier Wellen sollen neu zugeschnitten und die Kooperation mit anderen öffentlich-rechtlichen Anstalten verstärkt werden. Die beiden bisher eigenständigen Hörfunkwellen „Radio Bremen Melodie“ und die „Hansawelle“ werden zu einem Regionalprogramm „Bremen Eins/Hansa Melodie“ zugesammengefasst.

Über das dritte Programm B 1 des Senders Freies Berlin (SFB) startet am 1. Juni das Szene-Magazin „Berlin-Beat“, das der SFB zusammen mit dem privaten Musikkanal Viva finanziert und in Auftrag gegeben hat. Das halbstündige Programm wird auf Viva um Videoclips auf eine Stunde Programm ergänzt und wiederholt. Die beiden Veranstalter wollen damit neue Formen der Zusammenarbeit zwischen öffentlich-rechtlichen und privaten Anbietern ausprobieren. 
ZDF-Intendant Stolte kündigt am 30. Juni an, dass der Elektronische Programmführer (EPG) des ZDF an die so genannte d-Box angepasst worden sei und im August auf Sendung gehe. Mit der Anpassung an die von der Kirch-Gruppe eingeführte d-Box kann das ZDF nun mit einem eigenständigen Zusatzinformationsservice die bisher etwa 800.000 im deutschen Markt verbreiteten d-Box-Digitalempfänger erreichen.

Neuer Programmgeschäftsführer des Kinderkanals von ARD und ZDF wird am 1. Juli Frank Beckmann.

Der von ARD und ZDF gemeinsam veranstaltete Ereignis- und Dokumentationskanal Phoenix verlegt seinen Sitz im August von Köln nach Bonn in das Gebäude des ehemaligen Bonner ZDF-Studios.

In einer Stellungnahme vom 28. August stellt die Deutsche Welle ihre Position zum Konzeptpapier des Beauftragten der Bundesregierung für Angelegenheiten der Kultur und Medien (BKM) dar. Grundsätzlich lehnt die DW ab, sich an der „politischen Überzeugungsarbeit“ im Sinne der Bundesregierung zu beteiligen, denn dies sei „mit den verfassungsmäßig verbürgten Rechten und Pflichten des öffentlich-rechtlichen Rundfunks nicht zu vereinbaren“.

Die Europäische Kommission habe keine Bedenken gegen die ZDF-Pläne, einen Medienpark als Erlebniszentrum einzurichten, teilt das ZDF am 30. August mit. Gegen die ZDF-Pläne hatten drei deutsche Vergnügungsparks beim Landgericht Mainz geklagt, da sie der Ansicht waren, das ZDF mache sich mit seinem Projekt, das mit Fernsehen im engeren Sinne nichts zu tun habe, des unlauteren Wettbewerbs schuldig. Das Gericht wies die Klage am 26. Mai ab, weil es der Ansicht war, der ZDF-Medienpark sei sowohl unter rundfunkrechtlichen wie auch unter wettbewerbsrechtlichen Kriterien ein zulässiges Instrument der Zuschauerbindung.

Am 14. Oktober wird bekannt, dass der Mitteldeutsche Rundfunk (MDR) durch Finanzspekulationen mit der Landeswährung Ecuadors 2,6 Mio. DM verloren hat. Das Finanzgebaren und die Outsourcing-Politik des MDR geraten unter massive Kritik von Rechnungshöfen und Politik. Die Gremien des MDR unterziehen die Anlagepolitik des öffentlich-rechtlichen Senders daraufhin einer kritischen Bewertung und verlangen für die Zukunft eine Abkehr von der bisherigen Strategie. Der Verwaltungsrat verabschiedet am 11. November ein Eckwerte-Papier, das in Zukunft hochspekulative Finanzanlagen ausschließen soll.

Am 6. November startet der Westdeutsche Rundfunk (WDR) in seinem Dritten Fernsehprogramm „Stadtfernsehen“ für Köln und Dortmund. Montags bis freitags von 17.45 bis 18.30 Uhr sollen die Sendungen „WDRpunktKöln“ und „WDRpunktDortmund“ über die Metropolen in Nordrhein-Westfalen berichten.

Der WDR wird am 29. November von der ARD-Hauptversammlung zur geschäftsführenden Anstalt der ARD ab 1. Januar 2001 bestimmt, WDR-Intendant Fritz Pleitgen wird damit neuer ARD-Vorsitzender und Nachfolger von Peter Voß, Intendant des Südwestrundfunks.

Am 9. Dezember geht der ZDF-Theaterkanal mit einem programmbegleitenden Informationsangebot ins Internet.

Der Intendant des Saarländischen Rundfunks (SR), Fritz Raff, wird am 11. Dezember in seinem Amt bestätigt. Seine zweite fünfjährige Amtszeit beginnt am 1. August 2001.

Am 19. Dezember unterzeichnen der SFB und der Ostdeutsche Rundfunk Brandenburg (ORB) eine Vereinbarung über die Neugestaltung der Radio-Kooperation. Ab dem 1. Januar 2001 sollen von den vier gemeinsam veranstalteten Programmen „Inforadio“ und „Radio Kultur“ unter der Federführung des SFB und „Fritz“ und „Radio 1“ unter der Federführung des ORB betrieben werden. 
$\mathrm{Zu}$ Weihnachten strahlt das ZDF als Pilotprojekt erstmals vier Sendungen, zwei Krimis und zwei Fernsehfilme, mit türkischen Untertiteln aus.

\section{Privater Rundfunk}

\subsection{Landesmedienanstalten}

Bei der Gesamtkonferenz der Direktoren und Gremienvorsitzenden in der Arbeitsgemeinschaft der Landesmedienanstalten wird am 28. März ein Papier zur „Medienregulierung und Programmaufsicht im privaten Fernsehen - ein Positionspapier der Landesmedienanstalten im Kontext der Diskussion über ,Big Brother“" verabschiedet. Danach soll die Tätigkeit der Landesmedienanstalten nicht nur auf die bloße Rechtsaufsicht reduziert, sondern auch programmliche Entwicklungen wie etwa „Big Brother“ sollten verstärkt reflektiert und bewertet werden.

Die Gemeinsame Stelle Jugendschutz und Programm der Landesmedienanstalten (GSJP) stellt am 12. Oktober ihren Halbjahresbericht vor: Von den 33 behandelten Einzelfällen stammten 16 aus dem Bereich Spiel- und Erotikfilme, bei 14 empfahl die GSJP der jeweils zuständigen Landesmedienanstalt, rechtsaufsichtlich tätig zu werden. Allein 10 der beanstandeten Erotikfilme wurden von RTL II ausgestrahlt. Auch das neue Format „Big Brother“, das vom 1. März bis 9. Juni 2000 ausgestrahlt wurde, war Gegenstand der Überprüfung. Nach intensiven Beratungen wurde der für RTL II zuständigen LPR Hessen empfohlen, zunächst nicht rechtsaufsichtlich tätig zu werden, da RTL II zugesichert habe, das Konzept nicht zu verschärfen und täglich für eine Stunde auf die Kameraaufzeichnungen in den Schlafräumen zu verzichten. Am 31. Oktober werden zwei Sendungen von TM3 und Premiere beanstandet.

Reiner Hochstein, Direktor der Landeszentrale für private Rundfunkveranstalter (LPR) Rheinland-Pfalz, stirbt am 7. September. Aus gesundheitlichen Gründen hatte er zum 31. Oktober vorzeitig in den Ruhestand gehen wollen. Nachfolger wird am 30. Oktober Manfred Helmes.

Die Gemeinsame Stelle Jugendschutz und Programm (GSJP) verabschiedet am 6. September ein Positionspapier zu Jugendschutz und Konvergenz. Hierin erheben die Landesmedienanstalten u.a. den Anspruch, nicht nur die Kontrolle des privaten Hörfunks und Fernsehens zu übernehmen, sondern auch für Angebote im Internet und andere elektronische Mediendienste.

\subsection{Private Hörfunkveranstalter}

Der Vorstand der Landesanstalt für Kommunikation Baden-Württemberg (LfK) lizenziert am 28. Februar mit „www.chart-radio.de“ das erste ausschließlich über Internet verbreitete Radioprogramm.

Einen Tag vor Eröffnung der Expo 2000 in Hannover geht am 31. Mai mit „Radio 21 - Der neue Rocksender“ das dritte private landesweite Hörfunkprogramm Niedersachsens auf Sendung.

Am 10. Juni geht das „Dom Radio Köln“ auf Sendung. Das Soft-AC-Musikformat umgibt werktags fünf Wortleisten mit Informationen, Talks und Service sowie religiösen Sendungen. Das Programm ist bundesweit über Satellit und über das Kölner Kabelnetz zu empfangen und wird aus dem Etat der Hauptabteilung Bildung und Medien des Erzbistums Köln finanziert.

Am 10. November erhält der private Hörfunksender „Sunshine Live“ von der LfK 
eine besondere, bundesweit gültige Lizenz, mit der sich der Sender bundesweit um die Einspeisung ins Kabel bewerben kann. Das Programm ist bereits über Satellit zu empfangen.

Am 20. November geht in Berlin „FAZ 93,6 Berlin - Das Business Radio“ auf Sendung. Das Programm, das von der FAZ-Tochter Electronic Media veranstaltet wird, richtet sich - wie seine Vorgänger auf der gleichen Frequenz, „Newstalk“ und „Berlin aktuell“ - an die so genannte Wirtschaftselite.

Sechs Beiträge der Programmrubrik „Lach- und Sachgeschichten“ des sächsischen Senders „Radio Energy“ werden am 20. November vom Medienrat der Sächsischen Landesanstalt für privaten Rundfunk und neue Medien gerügt. Die Sendungen hätten u.a. durch verächtliche Äußerungen gegenüber Behinderten die Menschenwürde verletzt.

\subsection{Private Fernsebveranstalter}

Der Einkaufssender HOT teilt am 13. Januar mit, dass er im Geschäftsjahr 1999 erstmals Gewinn gemacht und ein Ergebnis vor Steuern in Höhe von über 20 Mio. DM erwirtschaftet habe. Der Bruttoumsatz aus Teleshoppinggeschäften sei im Vergleich zum Jahr 1998 um 75 Prozent von 199 Mio. auf über 350 Mio. DM gesteigert worden.

Am 24. Januar geht der neue Nachrichtenkanal der Pro Sieben-Gruppe, N 24, auf Sendung. Tags zuvor hatte N 24 sein Internet-Angebot gestartet. Der neue TV-Sender mit dem Schwerpunkt Nachrichten und Wirtschaft ist technisch nur in weniger als 30 Prozent der Haushalte via Satellit Astra 1 D sowie vor allem in Bayern über die Kabelnetze und Antenne zu empfangen.

Der Pay-TV-Sender Premiere World startet am 1. März ein an ältere Zuschauer (45+) gerichtetes Paket „Gala World“, das fünf TV-Programme mit deutschen und internationalen Schlagern, Filmklassikern und Heimatfilmen sowie Krimis und Dokumentationen umfasst. Das Paket kostet monatlich 19,90 DM plus Decodermiete.

Am 1. März startet auf RTL II das neue Sendeformat „Big Brother“: Fünf Frauen und fünf Männer leben für 100 Tage in einem von zahlreichen Kameras beobachteten Container, parallel dazu können die Hausbewohner 24 Stunden live über das Internet beobachtet werden. Alle 14 Tage muss ein Kandidat das Haus verlassen, wobei die Entscheidung darüber, wer zu gehen hat, das Publikum per TED-Wahl trifft. Der Sieger erhält ein Preisgeld in Höhe von 250.000 DM. Angesichts massiver Proteste gegen das neue Sendeformat, beschäftigen sich die Landesmedienanstalten mehrfach mit dem Programm. Die Gemeinsame Stelle Jugendschutz und Programm empfiehlt der zuständigen Aufsichtsanstalt LPR Hessen jedoch am 14. März, nicht rechtsaufsichtlich tätig zu werden. „Big Brother“ wird vor allem für die jüngeren Zuschauer zwischen 14 und 29 Jahren zum Fernsehereignis: Sie bescheren RTL II zur fraglichen Sendezeit durchschnittliche Marktanteile von 12-15 Prozent.

Ab 31. März wird das TV-Programm „Via 1 - Schöner Reisen“ im Basispaket des digitalen Telekom-TV-Angebots Media Vision angeboten. Da der zum Empfang nötige d-Box-Decoder noch keine direkte Rückmeldung über die Fernbedienung ermöglicht, müssen interessierte Zuschauer die Angebote des „Reise- und Teleshopping-Senders“ über Telefon oder Internet buchen. Bereits seit 21. Februar im Bouquet von Vision Select ist der Abenteuersportkanal „Extreme Sports Channel“.

Zum 31. März stellt RTL sein erst im September 1999 gestartetes morgendliches LiveMagazin „Mein Morgen“ wieder ein, da die Sendung keinen zufrieden stellenden Marktanteil erreichen konnte. 
Die Tele-München-Gruppe (TMG) verkauft am 26. April ihre 34-prozentige TM3Beteiligung an die News Corporation, die nun alleinige Inhaberin ist.

Der Kölner Musiksender VIVA Media AG geht am 19. Juli an die Börse.

Im Paket von Premiere World geht am 1. Oktober der Kindersender „Fox Kids“ täglich von 6.00 bis 22.00 Uhr innerhalb von „Family World“ auf Sendung. Fox Kids will die 3- bis 13-Jährigen erreichen. Das Programm ist teilweise werbefinanziert. Neben dem TV-Programm soll ein „Webauftritt mit Spielen, Chats und Aktionen“ angeboten werden. Der internationale Kinderfernsehveranstalter Fox Kids Europe gibt gleichzeitig bekannt, dass er aufgrund gestiegener Abonnentenzahlen und Erlöse die Rentabilitätsschwelle überschritten habe. Laut Pressemitteilung stiegen die Gesamterlöse um 27 Prozent auf 226 Mio. DM im Geschäftsjahr 1999/2000.

Am 31. Oktober kündigt die Hot Networks AG an, den Fernsehsender TM 3 übernehmen und zu einem Unterhaltungsprogramm machen zu wollen. Am 26. April war TM 3 zu 100 Prozent von der News Corporation Rupert Murdochs erworben worden. Die Hot Networks AG war Anfang des Jahres als Holding- und Management-Gesellschaft für den Aufbau einer europäischen Senderfamilie gegründet worden. Anteilseigner sind Georg Kofler und Thomas Kirch mit je 26,66 Prozent sowie das amerikanische Unternehmen Home Shopping Network (größter Anteilseigner beim Shoppingkanal HOT) mit den restlichen Anteilen.

Die Veränderungen in der Beteiligungsstruktur von SAT.1 werden von der KEK am 21. November als unbedenklich eingestuft. Die Veränderungen bei SAT.1 ergaben sich aus der Verschmelzung mit der Pro Sieben Media AG zur Pro Sieben SAT.1 Media AG. Als ebenfalls unbedenklich werden die Veränderungen bei den digitalen Pay-TV-Programmen Discovery Channel und Goldstar TV bewertet. An beiden Sendern hält die Multichannel GmbH, eine Beteiligungsgesellschaft der Kirch Pay-TV GmbH \& Co. KGaA nun 50 Prozent der Anteile. Grünes Licht gibt die KEK auf der gleichen Sitzung für die Onyx $\mathrm{GmbH}$, die künftig als Lizenzinhaberin von insgesamt 13 digitalen $\mathrm{Mu}$ sikspartenkanälen auftreten kann (5 von Onyx TV, 8 von Groupe AB S.A.).

Quellen: epd medien, Funkkorrespondenz, Media Perspektiven, DocuWatch Digitales Fernsehen, Medienspiegel, Presseinformationen der Sender und Landesmedienanstalten, eigene Recherchen 